\title{
A Vibration-Isolated Body of Chaotic Prediction
}

\author{
Yongyi Gao", a, Shiping Zhan ${ }^{1, b}$, Bangyan $\mathrm{Li}^{1, \mathrm{c}}$ \\ ${ }^{1}$ College of Physics, Hunan University of Science and Technology, Xiangtan 411201, China \\ agaoyongyi5188@163.com czspo401.love@163.com cfxxwl@163.com
}

Keywords: vibration isolator chaos, analytical prediction

\begin{abstract}
The nonlinear dynamics equation of passive vibration isolator is established in this paper. According to the nonlinear vibration theory, the average equation of slow-varying primary harmonic in the condition of weak nonlinearity is abstained, and derived a discrete mapping of the harmonic slow variable parameter state equation, then get the analytical conditions of chaos in the passive vibration isolator, the analytical results show that only when the vibration frequency of the groundsill is higher than the inherent frequency of the passive vibration isolator, the chaos can be observed, when the groundsill vibrate with the large amplitude and high frequency vibration, the chaos can't be observed in the passive vibration isolator system. Finally the analytical prediction is validated by analog simulation experiment, and gets the conclusion that the prediction matches well with the simulation results.
\end{abstract}

\section{Introduction}

In Project, in order to reduce the adverse effects of equipment that caused by vibration and guarantee the normal work, some measures of passive vibration isolator usually are taken to protect the precision instruments and devices in the work place. Vibration isolator materials are nonlinear, have non-linear characteristics [1-3]. It is difficult to take the theoretical analysis and calculation in the nonlinear vibration, so when it need to design and analyze the vibration isolation in project, it usually unreasonably omit the objective nonlinear factors in vibration isolation system, and the system will be simplified as a linear one. Therefore, study on the vibration problems of nonlinear vibration isolator has arise people's interest, and some useful progress has been made in recent years through the responsible report. In reference [4], the transformed cubic polynomial function was used to represent the stiffness of nonlinear material, established the nonlinear dynamics equation of passive vibration isolator, analyzed the influence that the nonlinearity of stiffness did to the steady-state response amplitude, resonant angular frequency and the effective vibration isolation range. In reference [5], established the nonlinear dynamics equation of passive vibration isolation. Derived the frequency responded equation; Discussed the influence of nonlinear factors; Analyzed the vibration stability of vibration isolator, get the boundary equation between stable zone and unstable zone. Tang Jin Yuan modified the energy iteration method [7], that was used to analyze the nonlinear vibration problem of passive vibration isolator when considering nonlinear stiffness. But little report of chaotic vibration in passive vibration isolator is found. The analytical prediction of chaos is an effective method in qualitative study of chaos [8-9], such as the Melnikov method [10-13], but the stiffness of routine used vibration isolation material is asymptotic, so it's hard to predict by Melnikov method. In this paper, we establish the first harmonic solution of the system, work out the state equation which the parameter of harmonic solution should obey. Then equalize and discrete the state equation .Derived the condition when saddle point of the state equation that the parameter obeyed came out through the discredited dynamic theory, and find out the analytical condition that chaos of the passive vibration isolator came out ,which provides necessary theoretical basis to the isolation designing when considering the nonlinear factors .

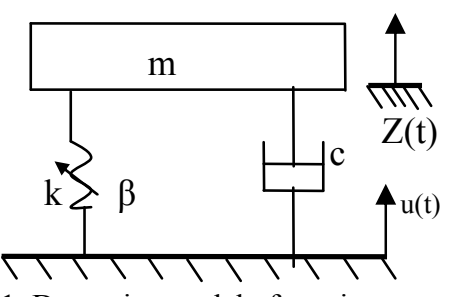

Fig. 1. Dynamics model of passive vibration isolator 


\section{Nonlinear dynamics equation of passive vibration isolator}

Figure 1 shows the dynamics model of passive vibration isolator. In reference [4], the author established the nonlinear dynamics equation of passive vibration isolator by using the transformed cubic polynomial function to represent the nonlinear stiffness of the material, as it shown in figure $1, \mathrm{Z}$ is the absolute displacement of the passive vibration isolator, $\mathrm{u}$ is the vibration displacement of the groundsill. According to the Lagrange Theory, the nonlinear dynamic equation of the passive vibration isolator when considering the nonlinearity of stiffness can be given as follows:

$$
m \ddot{Z}+c(\dot{Z}-\dot{u})+k(Z-u)+\beta(Z-u)^{3}=0
$$

$m$ is the mass of the passive vibration isolation object, $c$ is the viscous damping coefficient of the passive vibration isolation material, $k, \beta$ are stiffness coefficient of the spring.

$$
x=Z-u, u=u_{0} \cos \omega t
$$

$u_{0}$ is the swing of the vibration displacement, which can be measured. Drag (2) into (1), we get:

$$
\begin{aligned}
& m \ddot{x}+c \dot{x}+k x+\beta x^{3}=m \omega^{2} u_{0} \cos \omega t \\
& p_{0}^{2}=k / m, \quad \eta=\beta / m, \quad 2 \xi p_{0}=c / m, F=m \omega^{2} u_{0}
\end{aligned}
$$

Equation (3) turns into:

$$
\ddot{x}+2 \xi p_{0} \dot{x}+p_{0}^{2} x+\eta x^{3}=F \cos \omega t
$$

Equation (5) is another form of the nonlinear dynamic equation of passive vibration isolator when considering the nonlinearity of stiffness.

\section{Analytical predictions of chaos}

Harmonic's average equation of slow-varying parameter. According to the nonlinear vibration theory, when the nonlinear part is weak, the real motion is very close to the periodic motion, but the swing and the original phase change slowly with the time. And now, Harmonic Solution of the system equation (5) can be given as follows:

$$
x=a \cos \omega t+b \sin \omega t
$$

$\mathrm{a}$ and $\mathrm{b}$ are the slow-varying parameter which relate on time. Take the derivative calculation of equation(6), then get:

$$
\dot{x}=\dot{a} \cos \omega t-a \omega \sin \omega t+\dot{b} \sin \omega t+b \omega \cos \omega t
$$

Since $\mathrm{a}$ and $\mathrm{b}$ are the slow-varying parameter which relate on time, and then:

$$
\dot{a} \cos \omega t+\dot{b} \sin \omega t=0
$$

So, $\dot{x}=b \omega \cos \omega t-a \omega \sin \omega t$

$$
\ddot{x}=\left(\dot{b} \omega \cos \omega t-b \omega^{2} \sin \omega t-\dot{a} \omega \sin \omega t-a \omega^{2} \cos \omega t\right)
$$

Drag $\ddot{x}, \dot{x}, x$ into equation (5), then get:

$$
\dot{b} \omega \cos \omega t-\dot{a} \omega \sin \omega t=g(a b t)
$$

and

$$
\begin{aligned}
& g(a b t)=-2 \xi p_{0}(b \omega \cos \omega t-a \omega \sin \omega t)+\left(\omega^{2}-p_{0}^{2}\right)(a \cos \omega t+b \sin \omega t) \\
& -\eta(a \cos \omega t+b \sin \omega t)^{3}+F \cos \omega t
\end{aligned}
$$


From equation (8) and (11),we get:

$$
\left\{\begin{array}{l}
\dot{a}=-\frac{1}{\omega} g(a b t) \sin \omega t \\
\dot{b}=\frac{1}{\omega} g(a b t) \cos \omega t
\end{array}\right.
$$

Suppose : $\theta=\omega t$, take the average method to equation(13)in its right zone of $[0,2 \pi]$, then drag:

$$
\left\{\begin{array}{l}
\dot{a}=-\frac{1}{2 \pi \omega} \int_{0}^{2 \pi} g(a b t) \sin \theta d \theta \\
\dot{b}=\frac{1}{2 \pi \omega} \int_{0}^{2 \pi} g(a b t) \cos \theta d \theta
\end{array}\right.
$$

into the integration $g(a b t)$, then get the average equation:

$$
\left\{\begin{array}{l}
\dot{a}=-\xi p_{0} a+\frac{p_{0}^{2}-\omega^{2}}{2 \omega} b+\frac{3 \eta}{8 \omega}\left(a^{2}+b^{2}\right) b \\
\dot{b}=-\xi p_{0} b-\frac{p_{0}^{2}-\omega^{2}}{2 \omega} a-\frac{3 \eta}{8 \omega}\left(a^{2}+b^{2}\right) a+\frac{F}{2 \omega}
\end{array}\right.
$$

equation (15) is the harmonic's average equation of slow-varying parameter.

Discrete mapping of slowly varying parameter. Supposing

$$
\begin{aligned}
& U(a, b)=-\xi p_{0} a+\frac{p_{0}^{2}-\omega^{2}}{2 \omega} b+\frac{3 \eta}{8 \omega}\left(a^{2}+b^{2}\right) b \\
& V(a, b)=-\xi p_{0} b-\frac{p_{0}^{2}-\omega^{2}}{2 \omega} a-\frac{3 \eta}{8 \omega}\left(a^{2}+b^{2}\right) a+\frac{F}{2 \omega}
\end{aligned}
$$

Equation(15)can be written as:

$$
\left\{\begin{array}{l}
\dot{a}=U(a, b) \\
\dot{b}=V(a, b)
\end{array}\right.
$$

Supposing the equilibrium point of equation $(15)$ is $\left(a_{0}, b_{0}\right)$, then we get :

$$
\left\{\begin{array}{l}
0=U\left(a_{0}, b_{0}\right) \\
0=V\left(a_{0}, b_{0}\right)
\end{array}\right.
$$

Integral equation (18) in the range of $[0, T]$, since $a$ and $b$ have the characteristic of slowly-varying, then we get:

$$
\left\{\begin{array}{l}
a(T)-a(0)=\frac{2 \pi}{\omega} U(a, b) \\
b(T)-b(0)=\frac{2 \pi}{\omega} V(a, b)
\end{array}\right.
$$

take $U(a, b), V(a, b)$ of equation (20) for Taylor expansion near the point $\left(a_{0}, b_{0}\right)$, and use equation (19):

$$
\left\{\begin{array}{l}
a(T)-a(0)=\frac{2 \pi}{\omega}\left[A_{11} a(0)+A_{12} b(0)+E(a(0), b(0))\right] \\
b(T)-b(0)=\frac{2 \pi}{\omega}\left[A_{21} a(0)+A_{22} b(0)+F(a(0), b(0))\right]
\end{array}\right.
$$

in the above equations:

$$
A_{11}=\left.\frac{\partial U}{\partial a}\right|_{\substack{a=a_{0} \\ b=b_{0}}}=-\xi p_{0}+\frac{3 \eta}{8 \omega} 2 a_{0} b_{0}
$$




$$
\begin{aligned}
& A_{12}=\left.\frac{\partial U}{\partial b}\right|_{\substack{a=a_{0} \\
b=b_{0}}}=\frac{p_{0}^{2}-\omega^{2}}{2 \omega}+\frac{3 \eta}{8 \omega}\left(a_{0}^{2}+3 b_{0}^{2}\right) \\
& A_{21}=\left.\frac{\partial V}{\partial a}\right|_{\substack{a=a_{0} \\
b=b_{0}}}=-\frac{p_{0}^{2}-\omega^{2}}{2 \omega} b_{0}-\frac{3 \eta}{8 \omega}\left(3 a_{0}^{2}+b_{0}^{2}\right) \\
& A_{22}=\left.\frac{\partial V}{\partial b}\right|_{\substack{a=a_{0} \\
b=b_{0}}}-\xi p_{0}-\frac{3 \eta}{8 \omega} 2 a_{0} b_{0}
\end{aligned}
$$

$E(a(0), b(0)), F(a(0), b(0))$ are the higher order term of $a(0), b(0)$, the higher order term of equation (21) can be ignored near $\left(a_{0}, b_{0}\right)$,so:

$$
\left[\begin{array}{l}
a(T) \\
b(T)
\end{array}\right]=\left[\begin{array}{cc}
\frac{2 \pi}{\omega} A_{11}+1 & \frac{2 \pi}{\omega} A_{12} \\
\frac{2 \pi}{\omega} A_{21} & \frac{2 \pi}{\omega} A_{22}+1
\end{array}\right]\left[\begin{array}{l}
a(0) \\
b(0)
\end{array}\right]
$$

Obviously, equation (26) is the form of discrete mapping for the slowly varying parameter after several times iteration, just as:

$$
P(a(t), b(t))=T P(a(0), b(0))
$$

Comparing equation (26) with equation (27), we get that:

$$
T=\left[\begin{array}{ll}
\frac{2 \pi}{\omega} A_{11}+1 & \frac{2 \pi}{\omega} A_{12} \\
\frac{2 \pi}{\omega} A_{21} & \frac{2 \pi}{\omega} A_{22}+1
\end{array}\right]
$$

Form (28) is the Jacobian matrix of discrete mapping.

Analytical predictions of passive vibration isolator for chaos. Supposing $\lambda$ is the eigenvalue of T, so:

$$
\left(\frac{2 \pi}{\omega} A_{11}+1-\lambda\right)\left(\frac{2 \pi}{\omega} A_{22}+1-\lambda\right)-\left(\frac{2 \pi}{\omega}\right)^{2} A_{12} A_{21}=0
$$

According to the Discrete dynamics theory, we know that: when $\lambda_{1}<1<\lambda_{2}$, Harmonic slow variable parameter state equation of singularities of existence saddle points, slow variable parameter and over time just can appear chaotic motions, the system (5) will likely be a harmonic is chaotic movement, slowly- vary parameter $a$ and $b$ become chaotic with time goes by, the harmonic of system(5) may be chaotic motion, that is: when $\lambda_{1}<1<\lambda_{2}$, there may be chaos in system(5). The necessary conditions that chaos appears in passive vibration isolator can be given as follows:

$$
\left[\begin{array}{ll}
A_{11} & A_{12} \\
A_{21} & A_{22}
\end{array}\right]<0
$$

$\operatorname{drag} A_{11}, A_{12}, A_{21}, A_{22}$ into equation (30), then simplified, that is:

$$
\left(\xi p_{0}\right)^{2}+\left(\frac{p_{0}^{2}-\omega^{2}}{2 \omega}\right)^{2}+4 \frac{3 \eta}{8 \omega} \frac{p_{0}^{2}-\omega^{2}}{2 \omega}\left(a_{0}^{2}+b_{0}^{2}\right)+3\left(\frac{3 \eta}{8 \omega}\right)^{2}\left(a_{0}^{2}+b_{0}^{2}\right)^{2}<0
$$


There is parameter $a_{0}^{2}+b_{0}^{2}$ in equation (31), for omitting $a_{0}^{2}+b_{0}^{2}$, make $r_{0}=\sqrt{a_{0}^{2}+b_{0}^{2}}$, $\tan \theta_{0}=\frac{b_{0}}{a_{0}}$, then dragged into (19), that is :

$$
\left\{\begin{array}{l}
0=-\xi p_{0} r_{0} \cos \theta_{0}+\frac{p_{0}^{2}-\omega^{2}}{2 \omega} r_{0} \sin \theta_{0}+\frac{3 \eta}{8 \omega} r_{0}^{3} \sin \theta_{0} \\
0=-\xi p_{0} r_{0} \sin \theta_{0}-\frac{p_{0}^{2}-\omega^{2}}{2 \omega} r_{0} \cos \theta_{0}-\frac{3 \eta}{8 \omega} r_{0}^{3} \cos \theta_{0}+\frac{F}{2 \omega}
\end{array}\right.
$$

omit $\theta_{0}$, that is:

$$
\left(\frac{p_{0}^{2}-\omega^{2}}{2 \omega} r_{0}+\frac{3 \eta}{8 \omega} r_{0}^{3}\right)^{2}+\left(\xi p_{0} r_{0}\right)^{2}=\left(\frac{F}{2 \omega}\right)^{2}
$$

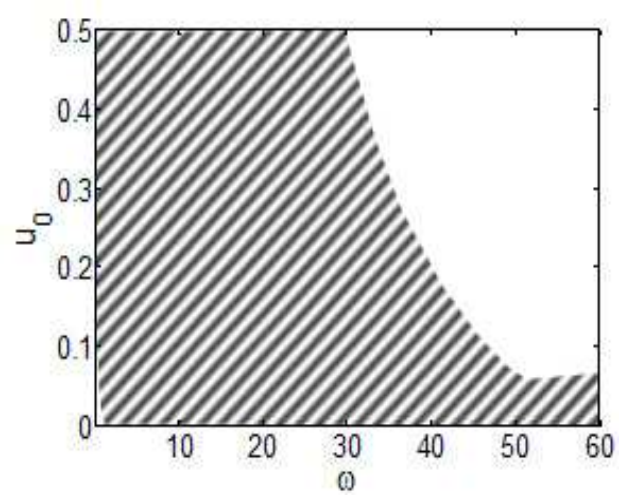

Fig. 2. Analytical prediction of chaos

Drag $r_{0}=\sqrt{a_{0}^{2}+b_{0}^{2}}$ into equation (31), that is :

$\left(\xi p_{0}\right)^{2}+\left(\frac{p_{0}^{2}-\omega^{2}}{2 \omega}\right)^{2}+4 \frac{3 \eta}{8 \omega} \frac{p_{0}^{2}-\omega^{2}}{2 \omega} r_{0}^{2}+3\left(\frac{3 \eta}{8 \omega}\right)^{2} r_{0}^{4}<0$

Equation (34) is the necessary condition that chaos appears in passive vibration isolator. Parameter $r_{0}$ in the equation can be represent by the system parameters, the function of $r_{0}$ and system parameters can be given by equation (33). Because of the fact that the nonlinear stiffness of the system will be greater when the value of $\beta / k$ and $\eta$ are larger, for making system (5) closer to weak nonlinearity, take the system parameters $\beta / k=0.4$ (the stiffness of the real used vibration isolator material for passive vibration isolator is asymptotic, here $\beta>0), \eta=0.4$, $m=1000 \mathrm{~kg}, \xi=0.2$. Draw the image of equation(34) by MATLAB, $u_{0}$ for $\mathrm{y}$-coordinate , $\omega$ for horizontal coordinates, the boundary of chaos observed is shown in figure2. Known from the equation (34), if it come into existence, the following equation must come into existence, that is :

$$
4 \frac{3 \eta}{8 \omega} \frac{p_{0}^{2}-\omega^{2}}{2 \omega}<0
$$

Calculate from the formula above and get: $\quad \omega>\sqrt{\frac{k}{m}}$

Known from formula (36), Only when the vibration frequency of the groundsill is higher than the inherent vibration frequency of the passive vibration isolator, chaos can be found

\section{Simulated verification of the analytical prediction}

Figure. 3 is the phase image of the simulation for equation (5). And $\mathrm{x}$-abscissa denotes for the displacement, $\mathrm{y}$ - abscissa denotes for velocity $(y=\dot{x})$, the initial value of the system takes $(0.1$, 0.1 ). when $u_{0}$ and $\omega$ take the value that beyond the prediction zone of chaos, such as $u_{0}=0.008$, $\omega=0.2$, no chaos is found in the system. as it shown in figure 3 (a); when $u_{0}$ and $\omega$ take the value that within the prediction zone of chaos, such as $u_{0}=0.8, \omega=1.5$, chaos can be found in the system. as it shown in figure 3 (b). The analytical prediction of chaos in passive vibration isolator is verified to be true by the simulation, and the prediction and judgment for the vibration isolator designing when considering the nonlinear factors can be used to avoid the adverse chaotic motion.

\section{Conclusion}

Through the above analysis can get the following conclusions:

1. When groundsill vibrates with both low and high frequency, the chaos phenomena of the passive vibration isolator system is not easy to found. 
2. Only when the vibration frequency of the groundsill is higher than the inherent vibration frequency of the passive vibration isolator, chaos can be found in the passive vibration isolator system.

3. Chaos won't be found in passive vibration isolator when the groundsill vibrates with wide swing and high frequency.

4. The simulation results and the analytical prediction results tallies well. So when adopting measures of passive vibrate isolator, the characteristic of the material and the external condition must be considered. Design as equation (34) shows, then the potential chaos can be avoided.

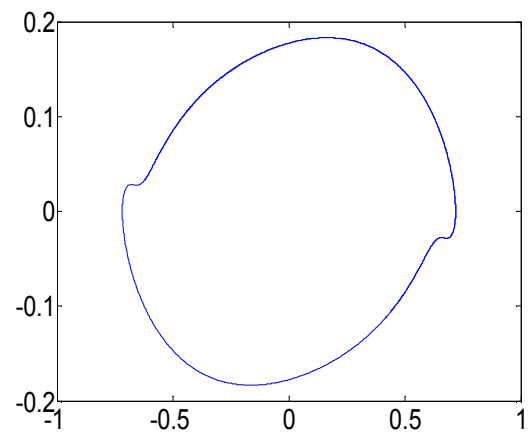

(a) $\omega=0.2, u_{0}=0.008$

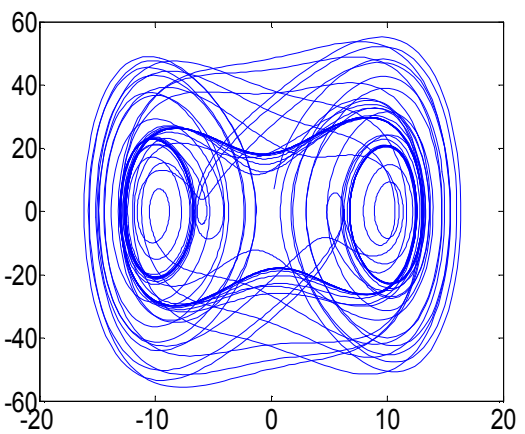

(b) $\omega=1.5, u_{0}=0.08$

Fig. 3. Phase images for different parameter

\section{References}

[1] Zhang Y H, Liu H H, Wang D C. Spring Guide, Beijing, 1997.

[2] Ertas A, Chew E K. Non-1inear dynamic response of a rotating machine. International Journal of Non -linear Mechanics, 1990, 25(23): 241-251.

[3] Mallik A K, Kher V, purl M. On the modeling of nonlinear elastomeric vibration isolators. Journal of Sound and Vibration. 1999, 219(2): 239-253.

[4] Chen A H, Liu D S, Zhu P Y. Nonlinear response analysis of passively vibration-isolated body,J. Chinese Journal of Mechanical Engineering. 2001, 37 (6) : 99-101.

[5] Chen A H, Gao W, Gao Y Y. Research on Nonlinear Dynamics of a Vibration-isolated Body, J. Chinese Journal of Mechanical Engineering. 2008, 19(9): 1022-1025.

[6] Tang J Y, Zhou Y F, He X H, Energy-iteration Method for Nonlinear Oscillation of a Passively Vibration-isolated Body, J. Chinese Journal of Applied Mechanics, ,2005,22(4): 621-622.

[7] Zhou Y F, Xie L H, Li L, Energy-iteration Method for Nonlinear Oscillation of a strong Nonlinear system, J. Chinese Journal of Applied Mechanics, , 1997, 14(4): 26-30.

[8] Zhou X,B Wu Y, Li Y, Hopf bifurcation analysis of the Liu system,J. Chaos Solitons \& Fractals, 2008; 36: 1385-1391.

[9] Forinash K, Willis C R. Nonlinear response of the SG breather to an a.c. driver,J. Physics D, 2001, 149: 95-106.

[10]Liu Z R, The analytical method in the study of chaos,Shanghai,2002.

[11]Zhu Z Y, Cai L Y, Liu W T, Computation of Chaotic Oscillation Parameter in Electrical Power System Based on Melnikov Method, J. Proceedings of the Chinese Society of Universities, 2008,20(3):42-45.

[12]Niu Y J, Xu W, Rong H W, Chaos prediction in the Duffing-type system with non-smooth periodic perturbation and bounded parametric excitation, J. Chinese Physics, 2008,57(12):7536.

[13]Rong H W, Wang X D, Bifurcations of Safe Basins and Chaos in Softening Duffing Oscillator under Multi-Frequency Harmonic and Hounded Noise Excitation, J, Journal of Applied Mechanics, 2009, 26(2): 275. 